\title{
Ethical Issues of Social Media Usage in Healthcare
}

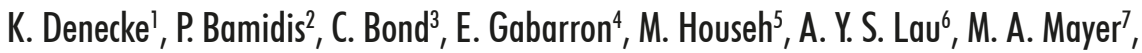 \\ M. Merolli ${ }^{8}$, M. Hansen ${ }^{9}$ \\ 1 University of Leipzig, Innovation Center Computer Assisted Surgery, Leipzig, Germany \\ 2 Aristotle University of Thessaloniki, Faculty of Health Sciences, Thessaloniki, Greece \\ ${ }_{3}^{3}$ Bournemouth University, School of Health and Social Care, Bournemouth, United Kingdom \\ 4 The Arctic University of Norway, Faculty of Health Sciences, Tromso, Norway \\ ${ }^{5}$ College of Public Health and Health Informatics, King Saud Bin Abdulaziz University for Health \\ Sciences, Ministry of National Guard - Health Affairs, Riyadh, Kingdom of Saudi Arabia \\ 6 Centre for Health Informatics, Australian Institute of Health Innovation, Macquarie University, Sydney, Australia \\ 7 Department of Experimental and Health Sciences, Universitat Pompeu Fabra - IMIM (Hospital del \\ Mar Medical Research Institute), Barcelona, Spain \\ 8 University of Melbourne, Health and Biomedical Informatics Centre, Melbourne, Australia \\ 9 University of San Francisco, School of Nursing and Health Professions, San Francisco, USA
}

\section{Summary}

Objective: Social media, web and mobile technologies are increasingly used in healthcare and directly support patientcentered care. Patients benefit from disease self-management tools, contact to others, and closer monitoring. Researchers study drug efficiency, or recruit patients for clinical studies via these technologies. However, low communication barriers in socialmedia, limited privacy and security issues lead to problems from an ethical perspective. This paper summarizes the ethical issues to be considered when social media is exploited in healthcare contexts. Methods: Starting from our experiences in social-media research, we collected ethical issues for selected social-media use cases in the context of patient-centered care. Results were enriched by collecting and analyzing relevant literature and were discussed and interpreted by members of the IMIA Social Media Working Group. Results: Most relevant issues in social-media applications are confidence and privacy that need to be carefully preserved. The patient-physician relationship can suffer from the new information gain on both sides since private information of both healthcare provider and consumer may be accessible through the Internet. Physicians need to ensure they keep the borders between private and professional intact. Beyond, preserving patient anonymity when citing Internet content is crucial for research studies.

Conclusion: Exploiting medical social-media in healthcare applications requires a careful reflection of roles and responsibilities. Availability of data and information can be useful in many settings, but the abuse of data needs to be prevented. Presenving privacy and confidentiality of online users is a main issue, as well as providing means for patients or Internet users to express concerns on data usage.

\section{Keywords}

Social media, health care ethics, information science, patientcentered care

Yearb Med Inform 2015;10:137-47

http://dx.doi.org/10.15265//Y-2015-001

Published online August 13, 2015

\section{Introduction}

Due to improved possibilities and means to obtain information about diseases and treatments that go hand-in-hand with the development of social media and Internet technologies, patients are becoming more informed [1], and they increasingly want to be engaged in their care [2]. Social media are digital media and technologies that enable users to exchange information and to create media content individually or in community with others. This media is increasingly becoming a tool supporting healthcare processes, gathering and sharing information, bringing people together, and encouraging social networking and communication regarding health topics [3], and it supports in this way patient empowerment, i.e. it brings patients into the position to take control of their healthcare needs. The evolution of the Internet from a limited, technical resource, to today's dynamic "Web 2.0" where people are able to share information means increasing numbers of people living with a long-term condition are now putting personal health information into the public domain, including discussion boards, social network sites, blogs, videos and virtual environments [4].

\subsection{Patient-centered Care and Medical Social Media}

The phenomenon of social media and its increased importance in the private as well as in the public sector show there are many potentials even in healthcare settings enabling patient-centered care. In particular, individuals suffering from chronic diseases are using social media more and more to communicate with others, exchange information, and human experiences. Peer-to Peer healthcare is emerging as a source for patient information and support [5]. Patients, family members, and friends share personal medical information, receive emotional support, or request guidance and advice from healthcare professionals via social-media sites. Social networking communities and data sharing platforms support sharing experiences with conditions, symptoms, and treatment outcomes, but also enable to track personal health and be actively involved in one's own care coordination. For researchers, such data provide new opportunities to analyze observational data to confirm results from randomized trials [6]. Increasingly, social networks are being used to investigate adolescent and young adult behaviors and personality traits [7], as well as for data collection and education purposes. One application area in this context is the recruitment of patients for clinical trials based on social-media profiles or the exploitation of social-media data for epidemiological studies [8]. Beyond, physicians may use social networking to crowdsource answers to individual clinical questions. Researchers have found, based on the data posted on Twitter, they can detect and monitor disease activity, most notably disease outbreaks such as cholera 
and influenza [9, 10], but more recently, data about issues like headache appearance was collected from tweets [11].

These examples show patient-centered healthcare, social media, and the Internet are beginning to come together. Patient behavior has notably changed already and will increasingly influence healthcare delivery and research. A couple of ethical questions arise when it comes to the use of social media in healthcare settings. If you have a Facebook or MySpace page with 600 "friends" is that your private page, or a public document? What do researchers need to consider when developing monitoring applications for healthcare using social media? What do health providers have to consider with respect to ethical questions of social-media usage?

\subsection{Ethics in Healthcare}

Ethics is defined as the discipline dealing with what is good and bad and with moral duty and obligation [12]. Public health ethics deal with the specific moral questions regarding public actions for disease prevention, life elongation, or psychological and physical well-being. This is in contrast to medical ethics which concentrates on the relationship between patients and doctors. The issue of how ethical principles may be applied to online health research is a current challenge for researchers, but also for health professionals and patients alike. In this paper, we start to explore these questions and topics.

\section{Objective and Methods}

The objective of this work is to examine the ethical implications of the aforementioned trends in the state of the art and to provide topics to be further addressed in the future. For this purpose, we selected use cases from our research work and analysis about the use of different social media platforms for health purposes. They include:

- the use of social media for the youth and the elderly,

- the impact on the patient-doctor communication and relationship,

- crowdsourcing in healthcare,

- the integration of social media in clinical environments,

- the use of wearable technologies, and

- research regarding medical social-media including harnessing patient-reported data, conducting online surveys and participant recruitment.

We collected and summarized ethical issues related to these use cases from our experiences and conducted a review of the literature (both white and grey). Further, we performed an environmental scan of popular and current applications and services in this area. The results were then discussed and interpreted. We concentrated on identifying and discussing relevant ethical aspects without writing a systematic review to match with the IMIA Yearbook objectives and structures. The authors of this work, all members of the IMIA Social Media Working Group (http:// www.imia-medinfo.org/new2/node/289), have skills and expertise to discuss around the results since we have a deep interest and a professional experience of using social media in healthcare. We have experienced ethical issues in our work.

\section{Ethical Issues of Social Media Usage}

\subsection{Use of Social Media for Youth}

There is a group of people who have grown up with the Internet: the youth, or the digital natives, regularly engage with new social-media [13], base their personal identities online [14], and social media is their natural environment. Youth is defined as a transition period from the dependence of childhood to adulthood's independence [15]. It is also the period when one's personal identity is formed, based on both individualities and the social environment [14]. For this digital native generation, the online social-media represents a space for connection, identity exploration, a space to express ideas, sexual identities, feelings, problems, and also a space where we receive feedback from others [16].
For a majority of youth, online social networking sites are their first point of call when they want to find information, including health-related matters $[17,18]$. Thus, social media is a rich environment to recruit youth participants to participate in research. For example, recruiting participants from Facebook and Twitter is one of the most effective recruitment strategies in youth-related research studies [19, 20].

Although using social media to recruit participants for research is positively viewed by the youth [21], it presents a number of ethical issues that need to be addressed. Obtaining informed consent from adolescents via social media represents a number of concerns [19]. Recruited participants over 18 years may provide their consent online, or their written consent, if they are redirected to a study site. But, how may we obtain parental consent from those contacted or interested under 18 years of age via social media [19]? In fact, is it ethical for study advertising material to be circulated in social media, targeting at those who are under-aged, and may have not reached the cognitive maturity to decide whether to participate or not? An alternative (and arguably more ethical) way to recruit very young people would be to target parents rather than children [19]. However, one needs to remember that policy settings in social media frequently change. For example, although Facebook reviews all the advertisements targeting young people under 18 years, their privacy policies can change without prior notice, and there is no mechanism to confirm one's age declared online.

When trying to reach youth through social media, whether it is for public health education or for public health monitoring, the same confidentiality and privacy rules that are applicable offline should also apply [22]. Although social media is a platform that allows a researcher to easily reach their targeted audience, establishing a dialog with them may not be as easy as one perceives. A recent qualitative study examining teenage patients' privacy concerns related to health matters revealed most participants did not disclose their personal health information on social media [17]. In this study, Facebook was seen to be a place for these teenage patients to be 'regular' teenagers, to stay 
up-to-date about their social life, and not seen as a place to discuss their diagnosis and treatment. In fact, the majority of them did not use social media to come into contact with others with similar conditions. Using social media as a health intervention needs to delicately consider participants' concerns for privacy. Some young users may prefer to interact with others anonymously, perhaps because they are struggling with sensitive issues, such as their sexual identities, or chronic diseases [17, 23]. Some would not want others to find out their true underlying health status and concerns, or they would not want updates about their health to be 'broadcasted' to their social network. On another scale, there are those who readily share very personal information that may be accessible by the broad general public [16]. In any case, young individuals' privacy and their level of comfort in discretion of health matters must be respected and preserved in the social media setting.

\subsection{Social Media to Train the Elderly and their Care Givers}

Like in the case of children and young people, elderly patients also have rights in the context of social media usage [24]. Despite recent discourse on whether the society should strive for technologic advancement of aging [25], or the related dispute between intrusiveness and isolation deepening with regards to elderly telecare/homecare [26, 27], this section touches issues related to the use of low-cost technology for elderly healthcare. Numerous efforts recently have been geared towards cognitive training or "brain training." The latter is usually marketed as a way for people to improve their memory and cognitive skills [28]. To this extent, the Internet and social media may be used as means of dementia (and other disease) prevention and health promotion [29] to train the elderly by cognitive exercises and novelty serious (web-based) games. For example, the training software Video GRade $[30,31]$ shows YouTube videos/documentaries to the elderly user (topics derived from nature, art and history etc.), and entails the user's attention control as it demands answers to simple questions at the end.
The aforementioned training piloting or deployment of the elderly through games and social-media content usually is confronted with the requirement of informed consent. This is highly associated with the certainty the affected party clearly understands what they are consenting to. Numerous studies have shown that whilst e.g. a checkbox may fulfill legal requirements to gather and record consent, it is not sufficient when the underlying ethical and environmental basis is that of elderly users, some of which may be mildly (cognitively) affected. Thus, future projects or systems could follow contemporary approaches to verify consent is both informed and relevant. This may be facilitated by suitable tutorial sessions and workshops on consent decisions and ensuring safe consent record keeping and processing. The consenting process could, in some cases, involve all interested stakeholders/parties: the patient, his/her care giver(s), and health professionals, in an effort to ascertain all parties understand what is being consented to.

Switching into a slightly different but still related theme, recent developments witness the use of the Internet and social-media content for training the elderly care giver(s) (formal or informal) [28]). For instance, the DISCOVER project aims at increasing carers' knowledge and independent digital skills development - as a way to enhancing their caring role - whilst at the same time providing a window to carers' needs for a range of service providers [32]. DISCOVER's ultimate aim is to improve the care giver's life quality, as well as the care and the life quality for those they care for. Usual ethical concerns in such projects, which often involve the conduction of relevant pilot trials, include those of confidentiality and privacy, consent, autonomy and choice, justice/fairness, inclusion, security, and dignity, with project guidelines not necessarily pointing to clear answers and possibly including conflicts between different ethical pointers. To accomplish care giver training, Internet content curation is one of the followed approaches enabled by means of appropriate curation tools like "Scoop.it!" and other project-based products. Within content curation, only carefully selected parts of content are considered and enriched with relevant commentary or insight, highlighting of important parts, and always assigning credit to the content's originator [33]. In this direction, one of the relatively unexplored issues is content curation ethics, with only a few recent attempts at establishing best practice guidelines available [34].

A last issue in supporting web training of care givers with social-media content is concerned with the ethical dilemma between certification and accreditation [35]. Currently, the use of social media better resembles "lightweight" rewarding process schemes rather than formal accreditation tactics and policies governed by regulatory bodies. However, the latter may be more effective in job hunting prospects. Current best practices consider that certification without accreditation is the most viable option, as it is contended to play an important role in increasing care givers' motivation to complete pilot training activities, as they promote a sense of satisfaction and reward, while enabling them to exhibit skills progression and reflecting commitment to personal development. Using Internet certificates and Internet badges to demonstrate non-accredited training reflects a growing global trend which might be a suitable ethical resolution to this problem until care givers reach any formal assessment points having consumed enough social-media content.

\subsection{Ethical Issues of Patient- doctor Communication through Social Media}

We already considered ethical issues related to social-media usage and research involving the elderly and the youth. In this section, we will look at the implications of social-media usage in traditional care settings, which involves patient-physician communication. Patient-physician communication in the traditional sense comprises the direct contact and questioning of the patient by the physician, and the discussion of treatment options. Information on diseases, therapies, and medications is exchanged; sometimes, administrational issues are clarified, such as making appointments. This communication is strongly characterized by medical confidentiality, trust, and privacy. Data is expected to be 
safely stored in the patient record, inaccessible to others, and even protected by law (e.g., in the Data Protection Directive 95/46/EC in Europe). With the development of Internet technologies, communication and monitoring in healthcare is starting to be outsourced to social media. Appointments can be made online, health information and even examination results can be distributed by e-mail. Social media can become an "icebreaker" that may improve the communication between patient and physician, resulting in better patient care [36]. However, this communication via the Internet is conflicted with a couple of ethical issues since technologies impact data privacy and security. Guidelines with respect to patient-doctor communications mainly address email communication [37, 38], and information exchange through websites, and concern confidentiality, unauthorized access to computers, informed consent, or privacy risks.

The patient-doctor relationship may suffer from two main situations. On the one hand, patients may have unrestricted access to their doctor's personal information as it is provided on the Internet. To address this issue, the American Medical Association (AMA) [39] recommends when using the Internet for social networking, physicians should use privacy settings to safeguard personal information and content or, even better, keep private and professional sectors separately. However, they should realize privacy settings are not absolute and once on the internet, content is likely to be there permanently. It is important for physicians and other healthcare professionals to familiarize themselves with the privacy provisions for different social-media applications and adjust the settings to ensure the content is clearly protected.

On the other hand, physicians have access to online patient information that may otherwise not be available in the healthcare setting (e.g. lifestyle information from patients posted in a personal blog). Such information about a patient received from online sources may be helpful in certain healthcare settings, but physicians need to be sensitive to the source and the way the information was displayed publicly. They should use their clinical judgment in determining whether and how to reveal such information during the treatment of patients. Digitally tracking the personal behaviors of patients, such as determining whether they have indeed quit smoking or are maintaining a healthy diet, may threaten the trust needed for a strong patient-physician relationship and have an influence on their treatment of the patient [5].

In summary, physicians must carefully maintain professional relationships and confidentiality in online settings. Emails and other electronic means of communication may supplement, but not replace, face-toface encounters. Establishing a patient-physician-online relationship, for example to "friend" a patient or ask a patient to "friend" a physician is ethically questionable [5]. The problem results from the fact the professional boundaries of interactions are less clear. Physicians may share personal, but also professional content online. Maintaining professional trust in a patient-physician relationship requires physicians to consistently apply ethical principles for preserving the relationship, confidentiality, privacy, and respect for individuals in online settings and mutual communications [40]. Online interactions with patients may pose challenges because of the ambiguity associated with written language without the context of body language or lack of awareness of the potential abuses of social-media data [5]. The AMA [39] also claims physicians should be aware of the standards of patient privacy and confidentiality that need to be maintained, and must refrain from posting identifiable patient information online.

\subsection{Crowdsourcing in Healthcare}

Another issue that influences the patient-doctor relationship is the opportunity for patients to seek answers to their healthcare questions through social media. Through crowdsourcing, patients can ask for a second or third opinion on a diagnosis or treatment of a medical condition. The "wisdom of the crowd" offers an opportunity to seek medical advice from other patients or clinicians from around the world through social networks such as Facebook, Twitter, HealthBoards, or Patients Like Me, and more recently, CrowdMed [41]. The term Crowdhealth referring to the use of social networks to solicit information from a large group of people on a treatment and diagnosis, or for a general health advice is new to healthcare. For example, in 2012, TIME magazine published the story of a husband seeking treatment for his ill wife by posting her health information on Facebook. He posted her medical records, symptoms, confirmed suspicions through his Facebook page and posted "I am throwing down all my cards on this one." [42]

With the growth of Crowdhealth, there are a variety of ethical issues related to the privacy of health information that are worth exploring since our current understanding of privacy and confidentiality are being challenged. Today, people are sharing their general and sensitive health information online looking for feedback from virtual community members. To illustrate, in 2011, a study on the sharing of sensitive health information through Facebook found Facebook users openly sought and shared information relating to behavioral, mental, and genetic health information [43]. The study explored publicly available Facebook groups and found users publicly identified themselves by divulging their name, photo, and location when seeking sensitive health information through online postings.

Furthermore, there is no contemporary healthcare privacy legislation in Europe or North America that may stop individuals from posting their health information online. The privacy laws in place today only protect an individual's healthcare information from institutions. Crowdhealth platforms, such as Crowdmed, clearly state on their Websites that "any medical information they provide will be published anonymously in the public domain, will be posted as-is, and it is the patient's own responsibility to remove any personally identifiable information from uploaded materials." [41].

Although the above statement is valid, the issue of healthcare privacy becomes murky when a parent shares their child's health information online along with pictures to a public audience, which was recently discovered on CrowdMed*. The posting has been removed, but the U.S. Children's Online

\footnotetext{
* The researcher conducted a search in July 2014, which found a Crowdmed patient posting their child's health information online along with a number of pictures of the child.
} 
Privacy Protection Act (COPPA) states on its Website, that an operator must obtain verifiable consent from the child's parent before collecting, using, or disclosing personal information from a child [44]. In this case, the parent posted their child's health information online, but issues concerning consent and how the information was posted remain unclear.

The ethical issues related to sharing health information in Crowdhealth platforms are challenging our perceptions on healthcare privacy. There are many ethical issues relating to how information is shared, how consent is obtained, relating to the use of the information for research or for commercial use, the sharing of information of minors by parents, and the validation of the user and the information they post. Crowdhealth, a new arrival, will only exacerbate these ethical issues relating to healthcare privacy.

\subsection{Integration of Social Media in Clinical Environments and Electronic Health Records (EHRs)}

Going along with the use of social-media tools in healthcare settings, there is a greater interest in considering the integration of social media in clinical environments as a source of health information. This is offering diverse opportunities for accessing health data and for the development of new research challenges [45]. There is an open debate about the way social media may be used as a tool to engage patients and professionals in healthcare, how health information is shared and posted in these platforms, the way social media should be managed in the context of a clinical information environment, and the potential benefits of including this information in patient records [46]. This integration includes the information provided by patients, as well as the interactions among professionals.

With people increasingly becoming involved in their own care and taking into account their patient experience, the idea of building comprehensive patient health records including social-media data and making this information accessible to the healthcare team gains in interest [47]. Pri- vacy is the basis of every patient-physician interaction, and protecting and safeguarding personal health data is not only an ethical imperative, but it is a legal requirement under different laws such as the Health Information Portability and Accountability Act (HIPAA) in the USA (Centers for Medicare $\&$ Medicaid Services reference), or the Data Protection Directive in the European Union (currently under review) [48]. Therefore, it is highly suggested they are applied to social-media interactions. In this context and as a reflection of the significance of the matter, the World Medical Association adopted a statement on the professional and ethical use of social media for health purposes in its last general assembly in 2011, urging national medical associations to establish guidelines for physicians addressing different issues related to social media [50].

Health providers and organizations will need to rethink how to collect and secure the data generated in social-media tools when including them in a clinical and EHR environment. At the same time, patients would need to be educated about what kind of information is protected as personal health information and what is not. In addition, improvements in privacy and confidentiality will be critical to foster the use of social media, and more experience is needed to determine its real clinical application [50].

Although the use of social media for health purposes and its inclusion in EHR are facing different unsolved questions, it is necessary to follow the same standards of quality and ethical issues that characterize health professions. There are several challenges that should be addressed, which affect aspects related to ethical and legal issues and operational aspects, as well as management of the information included in this new scenario. It is necessary to determine what kind of content related to social media can be considered as a piece of health information in a patient record, clearly establishing the mechanisms of control and the way that the elements related to social media are working integrated in an EHR environment, defining the use of policies applied to the context of social-media information. The appropriate use of social media channels for health purposes requires that health institutions and services provide patients with clear and simple recommendations to use social-media platforms when interacting with health professionals and at the same time, health professionals will benefit from specific training in using social media platforms both at undergraduate and professional activity levels.

\subsection{Wearable Technologies and Privacy of Data}

Wearable technologies, which include wearables and biosensors (see Figure 1), are currently receiving mixed reviews by research analysts [49]. However, this rising technology appears popular nowadays with an estimated 19 million items being shipped in 2014 and predicted purchases of up to 112 million by 2018 [51]. Grossman and Vella [52] explain how "wearable tech will change your life, like it or not (p.1)." Despite the mixed reviews and consumer "hype", the generated personal data provides a rich source of information posing ethical concern and generating ongoing discussion regarding privacy of data. The aim of this section is to present how wearable technologies could advance healthcare and determine the primary ethical issues in today's digital health ecosystem.

There are three different types of wearables: Fitness trackers and complex devices and applications, such as MyFitnessPal, for mobile devices to assist individuals with health promotion and maintenance (e.g. diabetes). Further, this technology can be used for the care of children, such as CryTranslator [53], and Sproutling [54], that assist caregivers in understanding why a baby may be crying (e.g. temperature changes or impositioning). Moreover, there is discussion around the cost benefits of wearables for those who have an existing medical condition (e.g. emphysema or congestive heart failure) since the data sent to the primary care physician may prevent hospitalizations [55].

Mobile medicine and technology come together with the ubiquitous smart accessories (smart phones, smart watches) requiring third party applications. For example, several tools for diabetes self-management exist, e.g. OnTrackDiabetes [56], Dbees [57], and Track3-Diabetes Planner 
[58]. Pain management and the use of smart phones by patients to record their pain events is assisting researchers to have a better understanding of women experiencing chronic widespread pain [59]. Thus, smart phones can be exploited to collect patient data on a moment-to-moment basis in order to receive more accurate data for clinical trials [60]. Third, autonomous smart wearables directly connect to the Internet, such as Google Glass or Vusix M100 SmartGlasses [61]. The almost hands-free monocle, Google Glass, enhances the users' experiences much like a smart phone device in the sense it has video capacity and a data retrieval system.

The combination of wearables with biosensors, such as activity trackers, smart watches, patches, and smart implants secure a wide range of physiological attributes, such as human sleep patterns, hydration, blood pressure, and glucose levels [62]. The increase in wireless access around the globe is making it easier to use wearable devices to monitor consumer wellness and assist with disease management. This explosive technology, displaying more of our body parts and personal activities online, is perhaps increasing the risk of sharing data where individuals may want to secure. Clearly, this leads to a major concern about access rights, security and privacy of this data. According to Trafford, the Food and Drug Administration (FDA) is taking steps to create guidelines as to what is 'considered' a medical device because applications for smart phones are not always a certified entity and may cause individual harm [60]. The live streaming firm, paired with Google Glass, CrowdOptic, reassures consumers there is no need to worry about personal privacy or Health Insurance Portability and Accountability Act issues because the company has devised a "lock down" system disallowing leakage of data. In May 2014, Google Glass was launched to the public. Glass "etiquette" has been developed and some public places are not allowing the use of the glasses due to privacy concerns [63].

Future discussion on the privacy concerns for this expanding market of wearables that literally provide your location, activity, and health data will be necessary. Individuals have to decide if they want to have wires

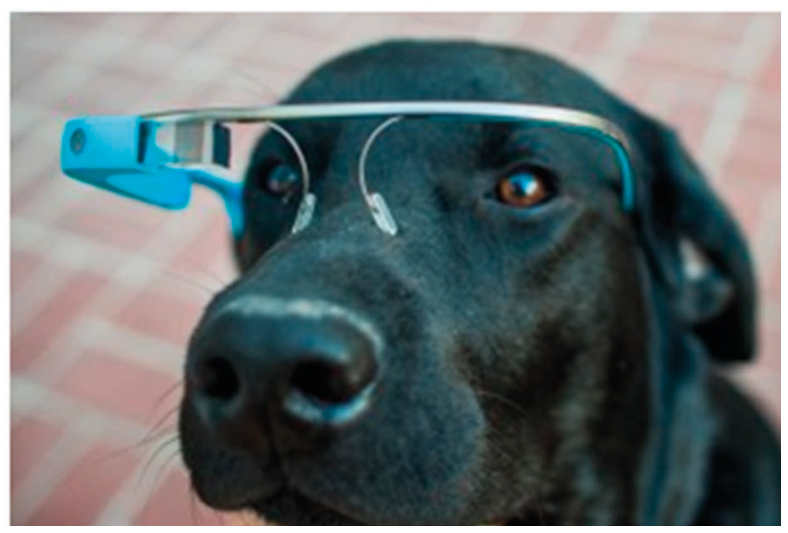

Fig. 1 Google Glass, Flickr Creative Commons, Thomas Hawk, May 2013

attached to them and their family members, as well as become more comfortable in sharing the wearable data included in their electronic records. Are we ready to allow the data obtained from wearables to be our guiding light in making health decisions? Perhaps with reassurance of increased data security and visible benefits gained from wearing body devices, we will become more trusting and motivated to use new technologies as they become more certified by the healthcare sector.

\subsection{Ethical Challenges of Using Social Media for Research: Harnessing Patient-reported Data, Online Surveys and Participant Recruitment}

\subsubsection{Using Peer to Peer Health Information as Research Data}

Usually in health research, the researcher decides both what will be asked and how, and participants choose how to answer the questions. That approach can be used online as well with the researcher posting questions and people deciding how (and indeed if) to answer them. It is also the easiest way to work out an ethical framework, as people may be told about how their replies will be used before they post them. The alternative is using material that people have already posted: rather than interviewees answering the researcher's questions, the 'participants' have put their views 'out there' for researchers to find, opening up a source of research data not obtainable elsewhere. This presents researchers with difficult decisions about the ethical framework to adopt, and the ways they will analyze and present the data. So far, there is a lack of consensus about the correct approach to use [64]. Whether this sort of research should be considered within a 'human subjects' framework or a more general humanities framework has been debated for over 10 years, e.g. [65. 66].

One voice that was missing in the debate is from people who contribute information through online discussion boards. A study was undertaken [58] to help address this gap. Using an asynchronous semi-structured qualitative approach, interviews were conducted with 30 people contacted through a discussion board for people with diabetes. Participants were aware that they had shared their information in a public domain, and there was a consensus that there was no problem with aggregated information (e.g. statistics or general trends) being used for analysis without consent. However, views diverged on using information with the potential to identify contributors. Some people felt if anything they had written was used then they should be acknowledged, whilst others thought their anonymity should be preserved. There was also disagreement on the question of consent; some people felt that their permission should be sought for the information to be used, whilst others did not feel this was necessary.

The Association of Internet Researchers (AoIR) agrees the concept of the human research subject is not a good fit with much 
online research, preferring to focus on practical issues such as harm, vulnerability, and identification. The study by Bond et al. [67] takes this further, identifying the merging of human subjects and online humanities has created a new type of research data, that of 'personal health text' which requires a new approach to ethics. The qualitative research norm of using quotes to support findings is particularly challenging when trying to also preserve anonymity whilst using online data. To address this Bond et al. [67] propose multiple quotes should be aggregated into a single aggregated quote that captures the essence of the original quotes, whilst not being searchable by search engines. Different types of online interactions may also require different approaches. People writing blogs and using their own names, promoted through media such as Twitter, might be more concerned with being cited whilst people writing under a pseudonym on a discussion board might be more concerned with anonymity.

\subsubsection{Online Surveys and Participant Recruitment}

Social media also offers the opportunity to recruit participants for clinical studies or online surveys. Online surveys offer research teams the ability to store large numbers of responses that are easily accessible for timely analysis [68]. Traditional recruitment methods (e.g., study brochures and telephone) are being surpassed by website posts and emails $[69,70]$. However, the emergent trend is social media, enabling even greater reach, visibility and dissemination of online surveys $[69,71]$. Recruitment may be quicker, more efficient and cost effective [70, 7274]. Consequently, as recruitment turns to social media, implications arise regarding ethical conduct [75].

Increased visibility is said to augment other recruitment efforts. According to Close et al. [69] and Yuan et al. [74], social media along with other web-based recruitment (e.g. email) 'layers' the recruitment message to help develop relationships. Ethically, this improves trust between target participants and researchers, as more opportunities to digest and consider participation in greater depth are provided. Ownership and increased autonomy over actual participation in a study are the results [69]. Similarly, it has been reported social media may help reach groups hard to contact in person [70-72, 74, 76]. This has positive implications for dealing with stigmatized and at risk populations, as greater levels of privacy and anonymity may be offered [74].

Conversely, online communities are a place to gather and work towards a common goal. The presence of outside entities is a sensitive issue and raises concerns regarding privacy. Similar issues were highlighted in 3.7.1 [68, 69]. Reaching out to vulnerable groups using social media poses a challenge to deal with any potential negative consequences. Care must be taken to neither disrupt the group, nor do any harm $[68,77]$. If group members sense study invitations aren't reflective of the community's core values and goals, barriers ensue as mistrust develops [69, 74]. According to Dyer [75], research should abide by medical codes of conduct, and online activities should not conflict with the principles of medical ethics.

Notably, the most reported ethical concerns for social-media recruitment are 'self-selection' and 'representativeness' $[71,72]$. Self-selection implies social-media users, especially those with an interest in the study area, will be recruited preferentially. Khazaal et al. [72] believe a further major consequence is that data will only represent certain participant's characteristics. Fox [78] discusses representativeness, identifying a skew towards well-educated and higher socioeconomic status cohorts online. Ethically, it may be argued these biases, coupled with coverage issues (e.g. lack of Internet access, poorer literacy), can negatively affect underserved populations and minority groups disproportionately absent online $[69,72,74,78]$.

The use of social media to recruit participants is forcing research institutions and ethical conduct committees to address fundamental areas of research ethics, such as: privacy, trust, autonomy, selection and representativeness. However, development of stringent methodologies is slow. Researchers should be encouraged by the potential of social media, but would be well served to ensure they use careful and rigorous research design.

\section{Future Trends}

Table 1 summarizes the ethical issues determined for the seven use cases. In this section, we will outline the future trends and relevant research aspects.

\subsection{Social Media and Patient Care}

It becomes clear from the previous sections that social media offer new possibilities for researchers, innovative ways for physicians to interact with patients, and that they positively affect the health outcomes of communities. However, the tenets of professionalism from all parties involved should govern these interactions. Health providers and organizations will need to rethink how to collect and secure the data generated by social-media tools when including them in a clinical and EHR environment.

In figure 2, we summarize the ethical aspects and issues to be considered. In the near future, social-media platforms could be reshaped and may be adapted as a tool for health communication among patients and professionals. There are some institutional policies on the use of social media, but no general policies are available yet. Maintaining a respectful and safe environment for patients, the public and physicians should be the main interest for all, researchers, physicians, and commerce. With respect to the patient-physician relationship, it is crucial to balance the information gained through the use of social-media technologies and the possible misuse or misinterpretation of data. Physicians are in principle able to conduct a social-media search for patients to learn more about their behavior or social circumstances. However, it is still unclear how to best use this information in healthcare and how it impacts the patient-physician relationship.

There are several negative aspects related to social media use. For example, it is used by the anti-vaccination lobby and the pro-anorexia online communities and can result in people getting into serious health problems. Analysis showed that there are open groups on Facebook for the promotion of healthy eating and in 
Table 1 Summary of ethical challenges for the seven use cases

\begin{tabular}{|c|c|}
\hline Use case & Lessons learnt / Challenges \\
\hline Use of social media for the youth & $\begin{array}{l}\text { - Obtaining informed consent of adolescents or their parents is problematic } \\
\text { - Young individuals' privacy and their level of comfort in discretions of health } \\
\text { matters must be respected and preserved }\end{array}$ \\
\hline Use of social media for the elderly & $\begin{array}{l}\text { - Suitable tutorial sessions on consent decisions might help to ensure an } \\
\text { informed consent is given from the elderly } \\
\text { - Care givers need to be trained and certified in social media usage } \\
\text { - Content curation ethics are still an open issue }\end{array}$ \\
\hline $\begin{array}{l}\text { Patient-doctor communication and } \\
\text { relationship }\end{array}$ & $\begin{array}{l}\text { - Physicians must carefully maintain professional relationships and confidential- } \\
\text { ity in online settings } \\
\text { - Establishing a patient-physician-online relationship is ethically questionable } \\
\text { - Patient data security and privacy need to be ensured }\end{array}$ \\
\hline Crowdsourcing in healthcare & $\begin{array}{l}\text { - Crowdsourcing raises considerable concerns regarding privacy, in particular, } \\
\text { when detailed health information from family members or friends are posted, } \\
\text { who are no longer able to give their informed consent }\end{array}$ \\
\hline $\begin{array}{l}\text { Integration of social media in clinical } \\
\text { environments }\end{array}$ & $\begin{array}{l}\text { - Social-media platforms should be reshaped and have to be adapted as a tool } \\
\text { for health communication among patients and professionals } \\
\text { - Patients, health professionals, and providers who are using these tools require } \\
\text { specific training }\end{array}$ \\
\hline Use of wearable technologies & - Data security, privacy and autonomy are major issues \\
\hline Social media for research purposes & $\begin{array}{l}\text { - Aggregation of quotes is acceptable by social media users when they become } \\
\text { subject of research } \\
\text { - Within patient recruiting from social media platforms, ongoing challenges are } \\
\text { trust between researchers and participants as social media research takes place in } \\
\text { the patient's environment and selection of participants should minimize biases. }\end{array}$ \\
\hline
\end{tabular}

fact around $40 \%$ of them promoted dietary products [79]. Further, it was found out that the majority of Facebook groups and vaccination groups (around 60\% in English and Spanish groups) were anti-vaccination groups [80].

Specifically, the elderly and children are at heightened risk of having activities or aspects of life monitored through social-media technology without consent. Furthermore, reports of these technologies as being "very intimate" do raise questions about the culture's view of the privacy of data that are captured, stored, and revealed [81]. Is there an ethical paradigm shift due to the addiction of the constant data stream? What are the views of healthcare professionals in regards to data privacy and human autonomy in today's evolving technological world? Do we need to have more privacy rules or should we forget about privacy issues? [81].

\subsection{Social Media and Health Research}

In patient recruitment, addressing privacy and mistrust from the presence of researchers and organizations is a big issue. Amongst ways to address or avoid this and limit any potential negative effects, refraining from contacting individuals directly is high on the list of suggestions. Dyer [75] iterates medical websites must ensure one's right to privacy is not infringed on. It would also be wise to avoid posting unscrupulous and pervasive recruitment messages on online communities without invitation. Involvement and funneling messages through group or page moderators is advised $[69,74]$.

The interpretive value of social-media data depends on the data analyses process. The data needs to be verified and corroborated with confirmed medical data to judge the interpretative value. The message resulting from self-selection and representativeness associated with using social-media cautions individuals to avoid generalizing study findings on an epidemiological level as data tends to represent a sub-group of the population [71, 72]. An important first step towards interpretable results is to determine the type of research being conducted and the target population. Researchers must first consider whom the research plans to target. When the population of interest is largely under-represented online, social media may not be the most appropriate channel $[71,68]$. Finally, in order to better understand the needs of under-represented and underserved populations, it may be useful for research to include examination of non-responders or a control group of non-internet users [72]. These ethical considerations for using social media to recruit survey participants continue to highlight the digital divide. Access to these technologies and education must remain high on the E-Health agenda to ensure underserved populations are afforded priority access and training to aid in health self-management.

A usual and yet unmet ethical challenge in piloting social-media content with elderly and young users is associated with the contemporary movement for "open data," where "open" means freely shared for anyone, anywhere, and to use for any purpose (see OpenDefinition.org). For example, the European Commission (EC) in the announced Horizon 2020 program promotes a pilot action on open access to research data thereby requiring participating projects to develop suitable data management plans, in which they specify what data will be open [82]. Two points should be considered at this end. First, when interaction with social-media content is recorded, perhaps de-sensitization of personal information is mandatory and the discussion held in other sections of this paper becomes relevant. Second, researchers should be aware of the recently released and relevant to this context Open Database License (ODbL, [83]) (instead of the usual Creative Commons - CC). ODbL allows unlicensed open data that is available and freely accessible (available online public data) for user downloads, 


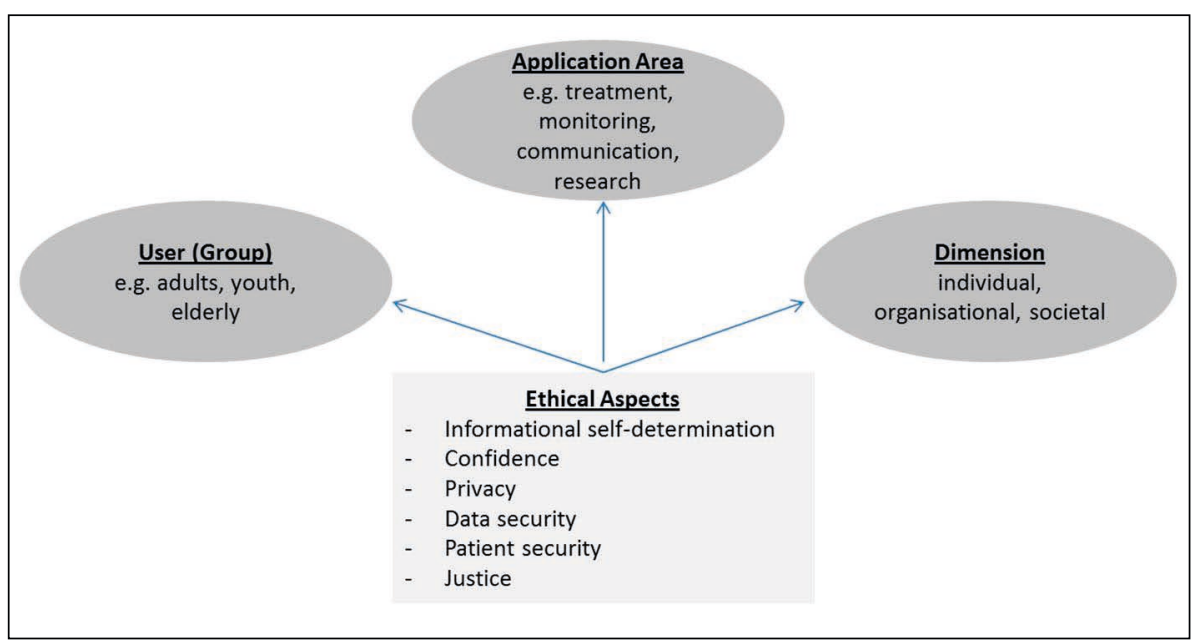

Fig. 2 Ethical issues of social-media usage in healthcare

copies, analyses, edits in any likely way, even with inclusion of data software; users should also be allowed to exploit them for any other purpose without financial, legal or technical barriers. This should now become the practice for proper digital rights management upon licensing of data and databases produced by research projects, government agencies or private institutions. The underlying ethical controversy between data openness and proper use of healthcare content interactions is obvious.

\section{Conclusions}

Preserving patient privacy and confidentiality in all environments is a main issue in the context of social-media usage in healthcare and research. This review of ethical issues tried to raise the important questions related to an appropriate use of social media in healthcare settings. Currently, there are still no official general guidelines available that may be applied to address these questions in practice. Given the broad application areas and involved stakeholders, it will be probably impossible to formulate general guidelines for all possible usage scenarios. For each application and research study, researchers and healthcare providers need to carefully weight harm and benefit for the individual patient or groups of patients. Such weighting could be supported by a novel model for systematical- ly evaluating technical solutions. In order to develop such model, relevant questions and with respect to the user groups, application areas and dimensions should be collected. The answers will help to judge and weight the ethical issues for technical solutions. In particular, future research study involving the use of social media should pre-plan in its design and pre-empt any ethically problematic effects introduced by its users, application area, and relevant dimension, and should develop sound solutions for addressing these ethical issues.

\section{Acknowledgments}

We acknowledge the IMIA working group members for their support and fruitful discussions.

\section{References}

1. Eysenbach G. Medicine 2.0: social networking, collaboration, participation, apomediation, and openness. J Med Internet Res 2008 Aug 25;10(3):e22.

2. Rozenblum R, Bates DW. Patient-centred healthcare, social media and the internet: the perfect storm? BMJ Qual Saf 2013.

3. Eckler P, Worsowicz G, Rayburn J. Social media and health care: an overview. PM R 20102(11):1046-50.

4. Armstrong N, Powell J. Patient perspectives on health advice posted on internet discussion boards: a qualitative study. Health Expect 2009;12(3):313-20.

5. Chretien KC, Kind T. Social media and clinical care: ethical, professional, and social implications. Circulation 2013;127:1413-21.

6. Wicks P, Vaughan TE, Massagli MP, Heywood J. Accelerating clinical discovery using self-reported patient data collected online and a patient-matching algorithm. Nat Biotechnol 2011;29: 411-14.

7. Ackland R. Social network services as data sources and platforms for e-researching social networks. Soc Sci Comput Rev 2009;27:481-92.

8. Brownstein C, Brownstein J, Williams D, Wicks P, Heywood JA. The power of social networking in medicine. Nature Biotechnology 2009;27:888 -90.

9. Denecke K, Krieck M, Otrusina L, Smrz P, Dolog P, Nejdl W, et al. How to exploit twitter for public health monitoring? Methods Inf Med 2013;52(4):326-39.

10. Collier N, Son N, Nguyen N. Omg U got flu? Analysis of shared health messages for bio-surveillance. J Biomed Semantics 2011;2(Suppl 5):S9.

11. Linnman C, Maleki N, Becerra L, Borsook D. Migraine tweets - what can online behavior tell us about disease? Cephalalgia 2013 Jan;33(1):68-9

12. Winter E, editor. Gabler Wirtschaftslexikon. Springer Fachmedien Wiesbaden, 18th Edition. Gabler Verlag; 2014.

13. Pew Research Center. Internet use over time. 2014. http://www.pewinternet.org/data-trend/teens/ internet-use/

14. Rafla M, Carson NJ, DeJong SM. Adolescents and the internet: what mental health clinicians need to know. Curr Psychiatry Rep 2014;16(9):472.

15. UNESCO 2014. What do we mean by "youth"? Available at: http://www.unesco.org/new/en/ social-and-human-sciences/themes/youth/ youth-definition

16. James C, Davis K, Flores A, Francis JM, Pettingill L, Rundle M, et al. Young People, Ethics, and the New Digital Media: A Synthesis from the GoodPlay Project. The MIT Press; 2009.

17. Van der Velden M, El Emam K. "Not all my friends need to know": a qualitative study of teenage patients, privacy, and social media. J Am Med Inform Assoc 2013;20(1):16-24.

18. Jain AV, Bickham D. Adolescent health literacy and the Internet: challenges and opportunities. Curr Opin Pediatr 2014;26(4):435-9.

19. Amon KL, Campbell AJ, Hawke C, Steinbeck K. Facebook as a Recruitment Tool for Adolescent Health Research: A Systematic Review. Acad Pediatr 2014;14(5):439-47.

20. Leonard A, Hutchesson M, Patterson A, Chalmers $\mathrm{K}$, Collins C. Recruitment and retention of young women into nutrition research studies: practical considerations. Trials 2014;15:23.

21. Moreno MA, Grant A, Kacvinsky L, Moreno P, Fleming M. Older adolescents' views regarding participation in Facebook research. J Adolesc Health 2012;51(5):439-44.

22. Denecke K. Ethical aspects of using medical social media in healthcare applications. Stud Health Technol Inform 2014;198:55-62.

23. Byron P, Albury K, Evers C. "It would be weird to have that on Facebook": young people's use of social media and the risk of sharing sexual health information. Reprod Health Matters 2013;21(41):35-44.

24. Perez-Carceles MD, Lorenzo MD, Luna A, E 
Denecke et al.

Osuna E. Elderly patients also have rights, J Med Ethics 2007;33:712-716.

25. Mallia P. Clinical intervention in aging: ethicolegal issues in assessing risk and benefit. Clin Interv Aging 2010;5:373-80.

26. Sorrel T, Drapper H. Telecare, surveillance, and the welfare state. Am J Bioeth 2012;12(9):36-44.

27. Welsh S, Hassiotis A, O'mahoney G, Deahl M. Big brother is watching you--the ethical implications of electronic surveillance measures in the elderly with dementia and in adults with learning difficulties. Aging Ment Health 2003;7(5):372-5.

28. Bamidis PD, Vivas AB, Styliadis C, Frantzidis C, Klados M, Schlee W, et al. A review of physical and cognitive interventions in aging. Neurosci Biobehav Rev 2014;44:206-20.

29. Miron-Shatz T, Hansen MM, Grajales FJ 3rd, Martin-Sanchez F, Bamidis PD. Social Media for the Promotion of Holistic Self-Participatory Care: An Evidence Based Approach. Contribution of the IMIA Social Media Working Group. Yearb Med Inform 2013;8(1):162-8.

30. Mcare LL. The Video Grade cognitive training platform, www.llmcare.gr, last access Oct 2014.

31. Frantzidis CA, Ladas AK, Vivas AB, Tsolaki M, Bamidis PD. Cognitive and physical training for the elderly: Evaluating outcome efficacy by means of neurophysiological synchronization. Int J Psychophysiol 2014 Jul;93(1):1-11.

32. DISCOVER project, Digital Inclusion Skills for Carers bringing Opportunities, Value and Excellence (project 297268) of the Competitiveness and Innovation Framework Programme Pilot actions by the European Commission. http://www.discover4carers.eu/, last access Oct 2014

33. Silva P. Content Curation for Online Education - Content curation as personal knowledge management - finding, gathering and organizing content with a critical perspective; 2012 . http://hyperallergic.com/51670/virginia-center-for-the-creative-arts-social-media-artists-fellowships/.http:// www.scoop.it/t/content-curation-for-online-students/?tag=ethics, Last update Dec 2012.

34. Deshpande P. Is Your Content Curation Ethical? A 10-Step Checklist. http://contentmarketinginstitute.com/2013/11/ethical-content-curation-checklist/, Nov 2013.

35. Bamidis PD, Sidiropoulos S, Vontas A, Wynne N, Waights V, de Graaf H, et al. Training Material and Quality Assurance Report - Deliverable D4.2 - Revision: 2.8 - Revised version of 1.6.3, DISCOVER project Deliverable, Access through www.discover4carers.eu

36. Payette MJ, Albreski D, Grant-Kels JM. "You'd know if you 'friended' me on Facebook": Legal, moral, and ethical considerations of online social media. J Am Acad Dermatol 2013;69:305-7.

37. Kane B, Sands DZ. Guidelines for the clinical use of electronic mail with patients. The AMIA Internet Working Group, Task Force on Guidelines for the Use of Clinic-Patient Electronic Mail. J Am Med Inform Assoc 1998 Jan-Feb;5(1):104-11.

38. eRisk Working Group for Healthcare's Guidelines for Online Communication https://www.acponline. org/running_practice/technology/comm_electronic.pdf [accessed: April 10 2015]

39. AMA (2014) American Medical Association. AMA Code for Medical Ethics. Opinion 9.124 -
Professionalism in the use of social media. URL: http://www.ama-assn.org/ama/pub/physician-resources/medical-ethics/code-medical-ethics/ opinion9124.page? (Access: 12.08.2014, 13:21).

40. Farnan JM, Snyder Sulmasy L, Worster BK, Chaudhry HJ, Rhyne JA, Arora VM, et al. Online Medical Professionalism: Patient and Public Relationships: Policy Statement From the American College of Physicians and the Federation of State Medical Boards. Ann Intern Med 2013;158:620-627

41. CrowdMed. Frequently Asked Questions. Does CrowdMed need to be HIPAA compliant (in the US)? [Date Accessed, September 8, 2014. https:// www.crowdmed.com/\#!/faqs]

42. Park A. "Web MDs: Social media are changing how we diagnose disease." Time 180.6 (2012): 16-16.

43. Househ M. Sharing sensitive personal health information through Facebook: the unintended consequences. Stud Health Technology Inform 2011;169:616-20.

44. COPPA - Children's Online Privacy Protection Act. [Date Accessed, September 8, 2014. http:// www.coppa.org/comply.htm]

45. Rodriguez-González A, Mayer MA, Fernández-Breis JT. Biomedical Information through the implementation of social media environments. J Biomed Inform 2013;46:955-6.

46. Herigon J. How social media will merge with electronic medical records. 2011 June 17. In: KevinMD.com. Social media's leading physician voice [cited 2014 Sep 25]. Available from: http:// www.kevinmd.com/blog/2011/06/social-media-merge-electronic-medical-records.html

47. Costa FF. Social networks, web-based tools and diseases: implications for biomedical research. Drug Discov Today 2013;18(5-6):272-81.

48. European Commission (EC). Justice. Protection of personal data. Reform of data protection legislation (2012) [cited 2014 Sep 25]. Available from: http:// ec.europa.eu/justice/data-protection/

49. Mayer MA, Leis A. How medical doctors and students should use Social Media: a review of the main guidelines for proposing practical recommendations. Stud Health Technol Inform 2012;180:853-7.

50. Moorhead SA, Hazlett DE, Harrison L, Carrol JK, Irwin A, Hoving C. A new dimension of health care: systematic review of the uses, benefits,and limitations of social media for health communication. J Med Internet Res 2013;15(4):e85.

51. Chen BX. Mixed predictions about wearables like smartwatches and Google Glass. Bits [Internet]. 2014 July 2 [cited 2014 Sept 11]. Available from: http://bits.blogs.nytimes.com/2014/07/02/ hot-or-not-smart-watches-and-google-glass/? $\mathrm{php}=$ true\&_type $=$ blogs\&_r $=0$.

52. Grossman L, Vella M. Never offline. TIME Magazine [Internet]. 2014 Sept 11. [cited 2014 Sept 19]. Available from: http://time.com/3326576/ never-offline/.

53. Translator App Decodes Crying Babies \& Tell you what is wrong. http://www.bitrebels.com/apps/ translator-app-decodes-crying-babies/ (accessed 16.04.2015)

54. Sproutling: http://www.sproutling.com/ (accessed 16.04.2015)

55. Going beyond mere fitness tracking: challenges and opportunities. http://elekslabs.com/2015/02/ going-beyond-mere-fitness-tracking-challenges-and-opportunities.html [accessed 28.04.2015]

56. OnTrack Diabetes - Android Apps on Google Play -https://play.google.com/store/apps/ details?id=com.gexperts.ontrack\&hl=en [last accessed: 15.04.2015]

57. Diabetes under control - dbees - http://dbees.com/ [last accessed: 15.04.2015]

58. Track3 Diabetes Planner -http://www.track3. com/android_diabetes_logbook/ [last accessed: 15.04.2015]

59. Kristjánsdóttir OB, Fors EA, Eide E, Finset A, Stensrud TL, van Dulmen S, et al. A smartphone-based intervention with diaries and therapist-feedback to reduce catastrophizing and increase functioning in women with chronic widespread pain: randomized controlled trial. J Med Internet Res 2013 Jan 7;15(1):e5.

60. Trafford E. Smartphones: A Better Approach To Clinical Trials. https://www.linkedin.com/pulse/ smartphones-better-approach-clinical-trials-evan-trafford (last accessed: 15.04.2015)

61. Subramanian C. Why 2014 is not the year of wearables [Internet]. 2014. [cited 2014 Sept 19]. Available from: http://fortune.com/2014/06/20/2014not-year-wearables/.

62. The future of biosensing wearables. Rock Health (2014). Slideshare presentation [Internet]. 2014. [cited 2014 Sept 11]. Available from: http:// rockhealth.com/resources/rock-reports/?gclid=CLiKmsjw5sACFUZbfgodYEcA9g.

63. Wessing, T. Introducing wearable technology [Internet]. 2013 June. [cited 2014 Sept 21]. Available from: http://www.taylorwessing.com/globaldatahub/article_intro_wearable_technology.html.

64. Whitehead LC. Methodological and ethical issues in Internet-mediated research in the field of health: an integrated review of the literature. Soc Sci Med 2007 Aug;65(4):782-91.

65. Eysenbach G, Till JE. Ethical issues in qualitative research on internet communities. BMJ $2001 \mathrm{Nov}$ 10;323(7321):1103-5.

66. Heilferty CM. Ethical considerations in the study of online illness narratives: a qualitative review. J Adv Nurs 2013;67(5),945-53.

67. Bond CS, Ahmed OH, Hind M, Thomas B, Hewitt-Taylor J. The conceptual and practical ethical dilemmas of using health discussion board posts as research data. J Med Internet Res 2013;15(6);e112.

68. Eysenbach G, Wyatt J. Using the Internet for surveys and health research. J Med Internet Res 2002;4(2):E13.

69. Close S, Smaldone A, Fennoy I, Reame N, Grey M. Using information technology and social networking for recruitment of research participants: experience from an exploratory study of pediatric Klinefelter syndrome. J Med Internet Res 2013;15(3):e48.

70. Ramo DE; Prochaska JJ. Broad reach and targeted recruitment using Facebook for an online survey of young adult substance use. J Med Internet Res 2012;14(1): e28.

71. Alshaikh F, Ramzan F. Social network sites as a mode to collect health data: a systematic review. 2014;16(7):e171

72. Khazaal Y, van Singer M, Chatton A, Achab S, Zu- 
Ethical Issues of Social Media Usage in Healthcare

1 lino D, Rothen S, et al. Does self-selection affect samples' representativeness in online surveys? An investigation in online video game research. J Med Internet Res 2014;16(7):e164.

73. Merolli M, Gray K, Martin-Sanchez F. Social Media and Online Survey: Tools for Knowledge Management in Health Research. In: Warren J, Gray K, editors. Proceedings of the Seventh Australasian Workshop on Health Informatics and Knowledge Management (HIKM 2014), Auckland, New Zealand, Conferences in Research and Practice in Information Technology (CRPIT) 2014;153:21-9.

74. Yuan P, Bare MG, Johnson MO, Saberi P. Using online social media for recruitment of human immunodeficiency virus-positive participants: a cross-sectional survey. J Med Internet Res 2014;16(5): el17.

75. Dyer KA. Ethical challenges of medicine and health on the Internet: a review. J Med Internet Res 2001;3(2):E23.

76. Bull SS, Levine D, Schmiege S, Santelli J. Re- cruitment and retention of youth for research using social media: Experiences from the Just/Us study. Vulnerable Childr Youth Stud 2012;8(2):171-81.

77. Fry C. Addressing the ethics of health eResearch with human participants. eResearch Australasia Conference, Melbourne, Australia. 2014.

78. Fox S. Chronic Disease and the Internet. Pew Internet \& American Life Project. Washington D.C.: Pew Research Centre; 2010.

79. Leis A, Mayer MA, Torres J, Rodríguez-González A, Suelves JM, Armayones M. Healthy eating support groups on Facebook: content and features (in Spanish). Gac Sanit 2013;(27):4.

80. Mayer MA, Leis A, Torres-Niño J, Rodríguez-González A. Information about Vaccines in English and Spanish on Facebook: Features and Content in Open Groups. iProceedings Medicine 2.0 London Sept 23-24, 2013:250-2.

81. Saffo P. Google Glass signals a wearables revolution. CNN Opinion [Internet]. 2014 April 16. [cited 2014 Sept 21]. Available from: http://www. cnn.com/2014/04/16/opinion/saffo-google-glass/.
82. EC, European Commission, Guidelines on Open Access to Scientific Publications and Research Data in Horizon 2020. http://ec.europa.eu/research/participants/data/ref/h2020/grants_manual/ hi/oa_pilot/h2020-hi-oa-pilot-guide_en.pdf, v1.0, Last update Dec 2013.

83. Murray-Rust P, Neylon C, Pollock R, Wilbanks J. Panton Principles, Principles for open data in science. Retrieved Oct 2014, from http://pantonprinciples.org

\section{Correspondence to:}

Kerstin Denecke

Innovation Center Computer Assisted Surgery

Semmelweisstr. 14

04103 Leipzig, Germany

Tel.: + 493419712002

Fax: +49341971 2009

E-mail: Kerstin.Denecke@medizin.uni-leipzig.de 